Volume 8, No.3, April - May 2019

International Journal of Wireless Communications and Networking Technologies

Available Online at http://warse.org/IJWCNT/static/pdf/file/ijwcnt13832019.pdf

https://doi.org/10.30534/ijwcnt/2019/13832019

\title{
Mental Disorders Detection by means of Online Social Media Mining
}

\author{
Anjana J Mani', Sruthy Emmanuel ${ }^{2}$ \\ ${ }^{1}$ Mangalam College of Engineering, India, anjanajmani333@gmail.com \\ ${ }^{2}$ Mangalam College of Engineering, India, \\ sruthy.emmanuel@mangalam.in
}

\begin{abstract}
Social Medias have turned into a piece of our lives. The broad utilization of social Medias may expedite an adverse effect in individuals' lives. In any case, our dependence via web-based networking media can detrimentally affect our psychological well-being. An expanding number of social media mental disorders (SMMD) have been observed recently. Mining on the social media gives a chance to effectively recognize SMMDs at a beginning period. In order to precisely recognize potential instances of SMMDs a machine learning framework is proposed, which exploits features extracted from social network data. The results show that the framework is promising for distinguishing the users with potential SNMDs at an early stage.
\end{abstract}

Key words: Feature Extraction, Machine learning, Mental disorder detection, Social Media.

\section{INTRODUCTION}

With the fast advancement of internet, individuals are progressively sharing their everyday lives .People love to connect with their friends through social networking communities. While Social Medias have their advantages, utilizing them also much of the time can make you feel progressively unhappy and confined over the long haul. The users never realize that their mental health is being affected by these Social Medias. Over the top utilization of existing social Medias is related with loss of time or disregard of essential drives and withdrawal, including sentiments of displeasure pressure melancholy when these social Medias are difficult to reach. Social Media doesn't care about user's mental well being.

A huge number of individuals every year experience the ill effects of dejection and just a division gets sufficient treatment. Identification of potential mental disorders often falls on the shoulders of teachers, parents. Studies show that 1 in 8 Americans suffer from problematic Internet use. The

American Journal of Psychiatry, have reported that the SMMDs may include excessive use of social media, depression, social withdrawal, and stress. To naturally recognize potential SMMD instances of OSN clients, removing these variables to survey clients' online mental states is testing. For instance, the degree of forlornness and the impact of Disinhibition of social media users are not actually noticeable. There are mainly six different ways in which social media affect us which includes mental health, memory, attention span, sleep, human connection.
Information overload and net compulsion are the two such social network mental disorders observed recently among the internet users [1]. Research in this area shows that there is a strong correlation between SMMDs and suicidal attempts[2], which point out that the teenagers suffering from social network addictions have a much higher risk of suicidal inclination. The behavior of users in social medias are observed and these symptoms are considered for diagnosis of SMMDs [3] .These symptoms constitute excessive use of social media apps which is associated with loss of time and the depression and tension a user experiences when these social medias are in accessible. A machine learning framework is developed which mines the data logs of online social network users. The user group affiliation information can be used to detect whether the user have any symptoms of SMMD. Recommendation in existing social media doesn't work well for cold users. Therefore it is necessary to analyze the Social and group information to improve recommendation accuracy for cold users than for heavy users.

\section{RELATED WORKS}

Nowadays people shows addiction towards internet just like as the addiction for alcohol and drugs. Internet Addiction Disorder (IAD) is a type of addiction observed in the world of Internet [4]. The consequences and correlations of IAD are discussed in many research works in the field of Psychology and Psychiatry[5][6][7]. The research in the area of social media regarding mental depression is of great importance and receives increasing attention [8],[9].From the user generated information content based textual features are extracted. Research shows that teenagers with shyness easily get addicted to Social Medias [10],[11].

Zhao proposed a progressive learning strategy for occasion discovery furthermore, determining by first removing the highlights from diverse information sources and after that learning through topographical staggered show. Be that as it may, the SNMD information from various OSNs might be inadequate because of the heterogeneity. The SMMD information from various social networks might be deficient because of the heterogeneity. In order to identify the bipolar disorder and borderline personality disorder Chang et.al employed an NPL based approach which collect and extract linguistic and content based features from social media. To analyze the pattern of activities of depression patients on social media sha[12] extracted the linguistic features. Major Depressive Disorder is observed by Choudhury [13] while analyzing emotion and linguistic styles of social media data. 
Most of the previous research mainly focused on individual behaviors and text contents generated by those individuals but the structure of social networks and potential Psychological features are not examined carefully. To overcome the drawbacks of previous approaches a multi-source machine learning approach, i.e., STM, is developed.

\section{PROPOSED SYSTEM}

The data available over social media through post, updates, status is a rich source for sentiment analysis and identifying users with SMMD. To reveal the online behaviors which are not covered in the traditional psychological criteria, Data analysis and case studies are conducted to offer more insights on how social media leads to mental health problems. The content and posting behavior of the users are analyzed to find out the characteristics of the depressed users. Users having stress and depression show similar emotions and they post contents related to death, anger and anxiety, failure, suicide.

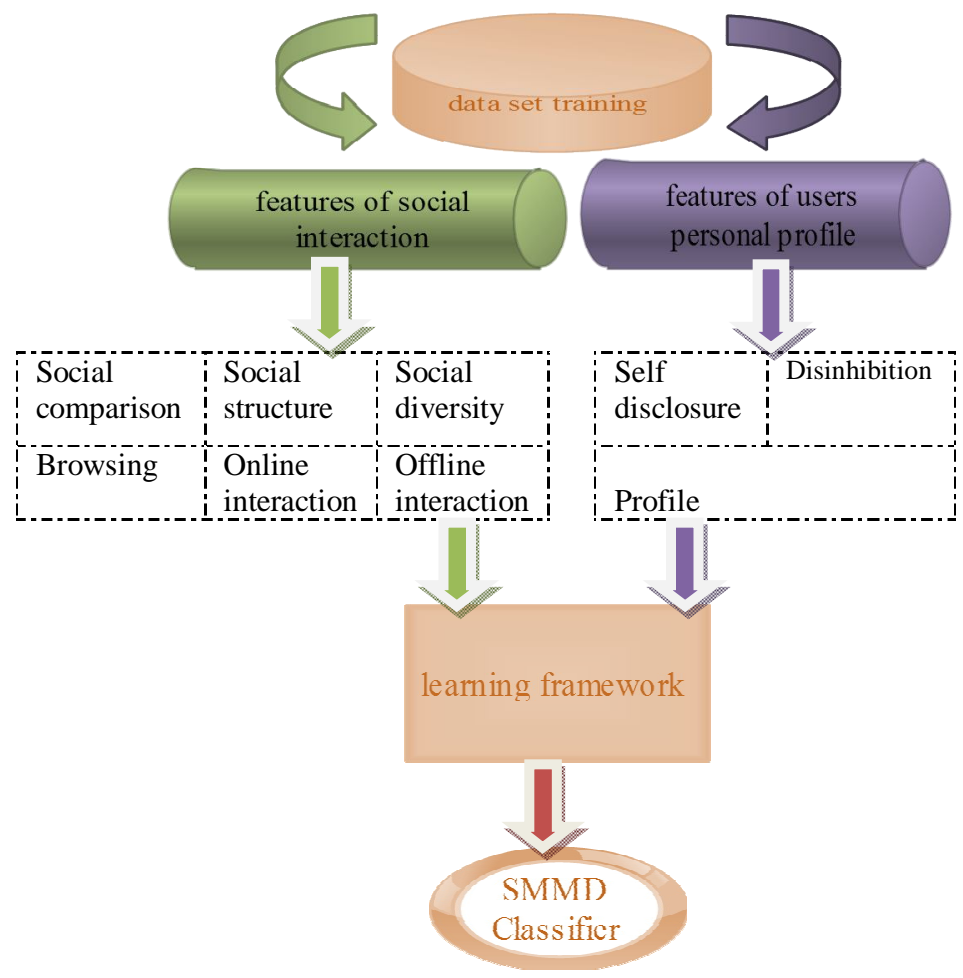

Figure1: SMMD detection framework

Thus by analyzing the contents of text posted by the users it is possible to detect whether the user is depressed or not. Not only the contents the users post but also the time at which they post may also contributes to the detection of depression. The depressed users usually bound to post updates somewhere in the range of 23:00 and 6:00, which mirrors that they might be vulnerable to a sleeping disorder. Machine learning systems can recognize the depression related words from peoples post on social Medias. Weka is a powerful machine learning tool that can be used for this reason and it gives more precise outcomes. The major advantage of social media is that it provides a platform for interpersonal interaction. Through these social media people get connected to each other from any part of the world.

Therefore its worth to analyze the correlation between users mental health status and fundamental social concepts, such that we may examine how and why a user's mental state is affected by other user. The figure1 shows the frame work which can be used to detect the behavior of users in social media. The point is to investigate information mining procedures to identify three kinds of SNMDs initial one is Cyber-Relationship (CR) Addiction, which incorporates the dependence on person to person communication, checking and informing to the indicate where social connections virtual and online companions turn out to could easily compare to genuine ones with loved ones, second one is Net Compulsion (NC), which incorporates urgent online social gaming or betting, frequently bringing about budgetary and work related issues; and third one is Information Overload (IO), which incorporates addictive surfing of client status and news sources, prompting lower work profitability and less social collaborations with families and companions disconnected.

\section{WORKING}

The SMMD framework observes the behavior of users in social media .It extracts the contents that are related to stress, depression and violence's from users comments, post, status updates etc. The user's group affiliation information is very useful in the context of social media mental disorder detection it helps to identify the interests of cold users. The cold users may not be active in updating their status or making comments on others post but they would be active in some groups. There are groups in social media which supports suicide, violence and anti social activities. By analyzing the activities in those groups it is possible to detect whether the user has any symptoms of social media mental disorder or not. This group affiliation information can also be used for giving social voting recommendation to users. Social voting is a feature of social media which enable a user to initiate voting campaigns on any topic.

The SMMD frame works on the basis of machine learning. Machine learning provides is used to automatically learn from experience without giving any program explicitly. The machine learning technique can be implemented in java by incorporating certain libraries. Weka is such a library that is used for data mining tasks in java. The algorithms in Weka can be called from the Java code of the SMMD system. These algorithms help to classify and cluster the user generated data. The user comments and post are classified by analyzing the contents. The SMMD check each and every user entries and decides whether a user is normal or have any social media related mental disorders .Not only the text content in social media but also the image contents are analyzed to check whether it is related to depression, stress,

violence or antisocial activities. OpenCv is a machine learning software library that can be used for this purpose. It contains 
several algorithms which can be used to detect and recognize the objects in the image. When the user uploads or shares an image the SMMD system analyze it because the users behavior and interest can be observed from the photos they like, post and share through social media.

\section{RESULT}

Online internet based life have turned out to be progressively well known in the course of the most recent couple of years as a methods for sharing diverse kinds of user created data such as, distributing individual announcements, transferring pictures, and sharing current location. User in a social media can likewise associate with different users by remarking on their posts and setting up discussions.

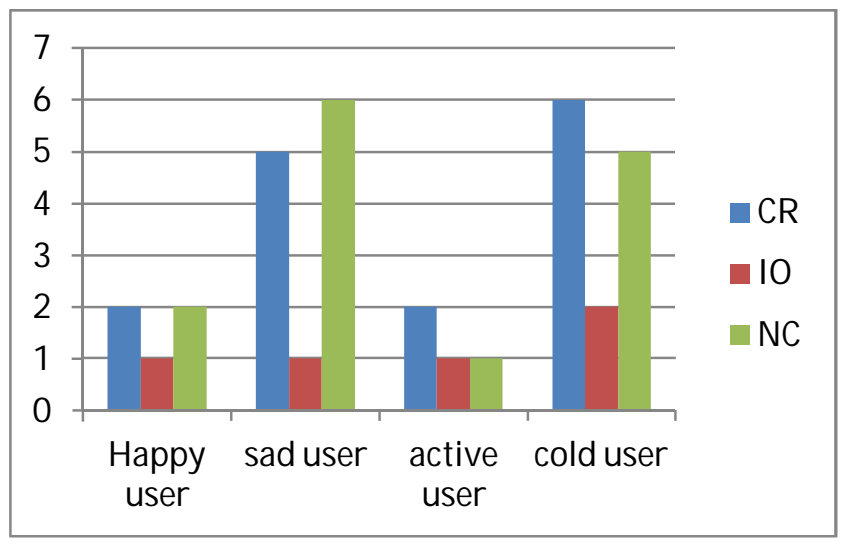

Figure 2: Graph showing mental status of users in social media

Through these associations, user can express their sentiments and considerations, and give an account of their every day exercises, making an abundance of valuable data about their social practices. The behavior of cold user, happy user, active user and stressed user is examined and find out various social media mental disorders such as cyber relationship addiction (CR),Net compulsion(NC),information Overload(IO).The observations show that the cold and sad users are more vulnerable to mental disorders (figure 2).

\section{CONCLUSION}

Social network mental disorder is a serious issue in our modern life. If SMMD is not diagnosed at early stage it will badly affect the user's life. A detection system can tackle this SMMD by monitoring user's behavior in social media by alerting the user if he/she tends to become a patient. These kinds of systems are necessary for the mental well being of people living in the world of internet. The increasing popularity of social voting brings forth the information overload problem. A user can be easily overwhelmed by various voting's that were initiated, participated, or retweeted by his or her direct and indirect friends. It is critical and challenging to present the "right votings"to the "right users". Analyzing the behavior of user in social media through machine learning will give a clear understanding about users likes and dislikes. This would be beneficial to provide social voting recommendations.

\section{REFERENCES}

1. K. Young, M. Pistner, J. O'Mara, and J. Buchanan. Cyberdisorders: The mental health concern for the new millennium. Cyberpsychol.Behav., 1999 https://doi.org/10.1089/cpb.1999.2.475

2. I.-H. Lin, C.-H. Ko, Y.-P. Chang, T.-L. Liu, P.-W. Wang, H.-C. Lin, M.-F. Huang, Y.-C. Yeh, W.-J. Chou, and C.-F. Yen. The association between suicidality and Internet addiction and activities in Taiwanese adolescents. Compr. Psychiat., 2014.

3. K. Young. Internet addiction: the emergence of a new clinical disorder, Cyberpsychol. Behav., 1998. https://doi.org/10.1089/cpb.1998.1.237

4. K. Young., Internet addiction: the emergence of a new clinical disorder Cyberpsychol. Behav., 1998. https://doi.org/10.1089/cpb.1998.1.237

5. Y. Wang. Linking multiple risk exposure profiles with adolescent Internet addiction: insights from the personcentered approach. Computers in Human Behavior, 2017.

6. K. Kim, H. Lee, J. P. Hong,M. J. Cho, M. Fava,D. Mischoulon, D. J. Kim, and H. J. Jeon. Poor sleep quality and suicide attempt among adults with internet addiction: a nationwide community sample of Korea. PLOS ONE, 2017 https://doi.org/10.1371/journal.pone.0174619

7. C.-H Chang, E. Saravia, and Y.-S. Chen. Subconscious crowdsourcing: a feasible data collection mechanism for mental disorder detection on social media, ASONAM, 2016. https://doi.org/10.1109/ASONAM.2016.7752261

8. B. Saha, T. Nguyen, D. Phung, and S. Venkatesh. A framework for classifying online mental health-related communities with an interest in depression. IEEE Journal of Biomedical and Health Informatics, 2016. https://doi.org/10.1109/JBHI.2016.2543741

9. M. Choudhury, M. Gamon, S. Counts, and E. Horvitz. Predicting depression via social media. ICWSM, 2013.

10. D. La Barbera, F. La Paglia, and R. Valsavoia. Social network and addiction. Cyberpsychol. Behav., 2009.

11. K. Chak and L. Leung. Shyness and locus of control as predictors of internet addiction and internet use. Cyberpsychol. Behav., 2004. https://doi.org/10.1089/1094931042403073

12. B. Saha, T. Nguyen, D. Phung, and S. Venkatesh. A framework for classifying online mental health-related communities with an interest in depression. IEEE Journal of Biomedical and Health Informatics, 2016. https://doi.org/10.1109/JBHI.2016.2543741

13. M. Choudhury, M. Gamon, S. Counts, and E. Horvitz. Predicting depression via social media. ICWSM, 2013. 